\title{
INVERSIÓN, CAPITAL Y PRODUCTIVIDAD EN ESTADOS UNIDOS, ESPAÑA Y BRASIL
}

\author{
Investment, Capital and Productivity in the US, \\ SPAIN AND BRAZIL
}

Juan Pablo Mateo Tomé

Universidad de Valladolid. Investigador del EMUI (España)

juanpablo.mateo@uva.es

Recibido: mayo de 2018; aceptado: octubre de 2018

\section{RESUMEN}

Este artículo muestra un análisis comparativo de la dinámica de acumulaciōn en tres economías con desigual nivel de desarrollo productivo, Estados Unidos, España y Brasil, durante el período 1995-2014, destacando las particularidades de los ratios del stock de capital y su relación con la productividad laboral. A pesar de que la evolución de las categorías de la acumulaciōn guarda ciertas similitudes, la investigación revela que estas economías adolecen de una cierta disparidad en la relación de los ratios capital-trabajo y capital-producto, logran un menor avance en la productividad laboral, y además, no se constata ninguna tendencia hacia la convergencia de España y Brasil con EEUU en términos de la productividad laboral en dólares.

Palabras clave: stock de capital, productividad, semiperiferia. 


\section{ABSTRACT}

This article shows a comparative analysis of the dynamics of accumulation in three economies with unequal levels of productive development, United States, Spain and Brazil, during the period 1995-2014, highlighting the particularities of the capital-stock ratios and their relationship with labor productivity. Despite the similarities in the evolution of the categories of capital accumulation, this research reveals that these economies suffer from a deep disparity in the relationship among capital-labor and capital-output ratios, have a lower increase in labor productivity, and furthermore, no tendency towards convergence of Spain and Brazil with the US in labor productivity in dollars can be grasped.

Key words: capital stock, productivity, semiperiphery.

Códigos JEL: E11, E22, E40, O47, O57. 


\section{INTRODUCCIŌN}

Este artículo muestra un análisis comparativo de la dinámica de acumulaciōn en tres economías con un nivel heterogéneo de desarrollo productivo, Estados Unidos (EEUU), España y Brasil, durante el período 1995-2014, y a partir de un enfoque de economía política. Específicamente, se estudia la relaciōn entre el stock de capital y la productividad, con el propósito de revelar las particularidades de cada economía y tratar de identificar si existe alguna tendencia hacia la convergencia en términos de productividad laboral.

Los conceptos teórico-abstractos de centro y (semi)periferia son apropiados por su carăcter ilustrativo y clarificador (véase Martínez, 2011), si bien no estān exentos de limitaciones, y sus fronteras pueden ser difusas. Aunque no se asume completamente el marco teórico estructuralista de raíz cepalina, y el enfoque circulacionista (Astarita, 2010), las categorías mencionadas resultan pertinentes para diferenciar - cualitativamente- diferentes grupos de países. El criterio seguido prioriza la esfera de la producción, a saber: desarrollo productivo y la capacidad de generar excedente de manera sostenida en el tiempo. Cuantitativamente, se refleja en variables como el PIB pc, la productividad laboral o el contenido tecnolōgico del stock de capital, junto aspectos de la estructura económica y, por extensión, en indicadores de ingresos, desigualdad y bienestar.

El desarrollo productivo se relaciona con la disponibilidad de activos de capital (K). Asī, el stock de capital constituye una variable central que en gran medida condiciona el tipo de evoluciōn macroeconómica en el tiempo y explica las diferencias en el nivel de desarrollo productivo entre los países. Se puede definir como una magnitud de valor inserta en un proceso de incremento de valor -valorizaciōn-, y materializado en el conjunto de elementos, tangibles o no, que forman parte del proceso, a saber, capital-dinero, capital productivo y capital-mercancía, es decir, se considera una relación social de producción (véase Mateo, 2007). ${ }^{1}$ Por ello, se plantean las siguientes preguntas: i) ¿cuál es la correspondencia entre el nivel de los ratios del capital y la productividad? ii) ¿cuáles son las particularidades de la evolución del capital respecto de la productividad en las fases de crecimiento y crisis? iii) ¿Cómo influyen los aspectos

1 Esta definiciōn no coincide con el fundamento teórico de las medidas oficiales, ya que, por una parte, abarca todos los elementos susceptibles de poseer valor, al margen de su contenido material, pero al mismo tiempo, se refiere sōlo al ámbito capitalista. Sobre ello, remito a Cámara (2003), Mateo (2007) y Shaikh (2016). 
monetarios en esta relación?; y por tanto iv) ¿cabe afirmar que existe una tendencia hacia la convergencia en términos de productividad?

En este documento, el término "centro" hace referencia a las áreas más desarrolladas, como de hecho son designadas por los propios organismos internacionales. La denominada "periferia”, por su parte, agrupa un conjunto de economías con mayor atraso, es decir, con bajos niveles de productividad, en general con una dependencia de actividades primarias con bajo contenido tecnológico, lo que lleva a un menor PIBpc, pobreza y menor índice de desarrollo humano. Entre estos dos grupos se puede situar una "semiperiferia", concepto que surge por los cambios acontecidos en la división internacional del trabajo en las últimas décadas (Martínez, 2011). Aunque no es de fácil delimitaciōn, hace referencia a aquellas economías que, sin ser parte del mundo desarrollado, tienen niveles de productividad y stock de capital superiores a las periféricas, se integran en algunas cadenas globales de valor, o poseen actividades con cierto nivel de desarrollo productivo, cuyos ejemplos más significativos serían México, Brasil, Argentina y Chile en América latina, junto al resto de integrantes de los BRIC y países recientemente industrializados de Asia. ${ }^{2}$

La elecciōn del período se deriva de aspectos relacionados con las economías de Brasil y España. Por una parte, el inicio en 1995 se explica porque en Brasil se aplica el Plan Real en 1993-94 para controlar la hiperinflaciōn, que ya en 1995 se encuentra en niveles reducidos, mientras que en España se ha implementado un programa económico que sigue las directrices de los acuerdos de Maastricht. Por otra parte, el estudio llega hasta 2014 debido a la disponibilidad estadística en Brasil, y además, en el caso de España existen series de cuentas nacionales consolidadas desde $1995 .^{3}$

Esta perspectiva analítica del proceso de acumulación es relevante, en primer lugar, en la medida que constituye la dimensión central que explica el proceso de crecimiento, por lo que incide sobre las esferas de la distribución, la monetaria-financiera y, así, sobre el crecimiento económico. Por el lado de la demanda, la inversión, y no el ahorro, es la variable fundamental para caracterizar los ciclos de expansión y crisis, y se materializa en un determinado volumen de capital. En segundo lugar, debido a la ausencia de estudios empíricos que incorporen economías con desarrollo dispar, los cuales, en todo caso, priorizan los flujos de inversiōn.

Y en tercer lugar, la pertinencia de este enfoque se justifica a su vez por las particularidades del contexto histórico y de los casos de estudio. EEUU, España y Brasil son economías con diverso nivel de desarrollo productivo. La primera representa la principal potencia económica, mientras que España,

2 Para esta clasificaciōn, remito a Martínez (2011), Palazuelos (2016), y sobre la problemática relativa a la semiperiferia, véanse Worth y Moore (2009).

3 Se ha de señalar que, en el caso de Brasil, ciertos ratios de la primera mitad de los años noventa ofrecen resultados anómalos debido a su hiperinflación, y además, sôlo se dispone de deflactor de la inversiōn hasta 2013. 
siendo una economía desarrollada perteneciente a la OCDE, está ubicada en la periferia de un área avanzada como la Eurozona (Del Rĩo, 2018), con un PIB pc que representa en torno al 59-65\% del estadounidense en 1995-2014. Brasil, sin embargo, es un ejemplo de economía semiperiférica con enclaves industrial y financiero (Palazuelos, 2015), integrando el grupo de las economías emergentes - los BRIC - y cuyo PIB pc es la quinta parte del de EEUU (Banco Mundial, 2017). En este sentido, las dos décadas de estudio se caracterizan por el giro hacia políticas económicas con cierto carácter neoliberal en España y Brasil, en las cuales la estabilidad monetaria y una apreciaciōn del tipo de cambio se convirtieron en aspectos distintivos. ${ }^{4}$ Es decir, se trata de un período en el que a priori se corrigen ciertas tendencias monetarias polarizadores que serān expuestas en el marco teórico, y que inciden de manera crucial sobre el modelo de acumulaciōn.

Para ello, el artículo comienza exponiendo los elementos teóricos del proceso de acumulaciōn y las asimetrías presentes en un esquema centro-periferia. Posteriormente, se procede a analizar los resultados del estudio empírico, primero en relación a la evolución de las diferentes categorías, y después llevando a cabo una comparativa de España y Brasil respecto de EEUU.

\section{AsPeCTOS TEÓRICOS}

\subsection{DiNÁMICA DEL PROCESO DE ACUMULACIŌN}

En primer lugar, ubicamos el objeto de investigación, la acumulaciōn de capital, en su marco más general. Desde un enfoque de economía política (Shaikh, 2016), el crecimiento del producto (Y) depende fundamentalmente de la inversión (I), la cual responde al objeto de maximizar el beneficio (B), por lo que se tiene la causalidad siguiente: $\{r \rightarrow I \rightarrow Y\}$, en la cual, no obstante, existe igualmente una reciprocidad en cuanto a la incidencia de las diversas variables. ${ }^{5}$ Así, la rentabilidad (r), que relaciona el objeto de la actividad económica (B) con el valor monetario del conjunto de elementos necesarios para obtener dicho rendimiento -y procedentes de los flujos de inversiōn-, el stock de capital (K), se puede expresar en función de tres tipos de ratios del capital:

$$
r=\frac{B}{K}=\frac{\left(\pi P_{y}\right)-\left(w_{L} P_{c}\right)}{\theta P_{k}}=\frac{e}{\tau}=\frac{\beta}{\delta}
$$

4 Se utiliza el término neoliberalismo porque se constata una diferencia con las fases anteriores en cuanto a la lógica que articula las decisiones fundamentales de política económica (vêase Mateo, 2016). Por supuesto, ello no implica una semejanza absoluta con el contenido del término, pero su pertinencia (no sin matices) para Brasil y España se puede verificar en Carcanholo (2011) y Buendía y Molero-Simarro (2018), respectivamente.

5 Por tanto, se entiende que la tasa de ahorro es una categoría derivada, pues no se explica a partir de una decisión libre, sino que responde a la necesidad de invertir y la capacidad de generar excedente. 
La tasa de beneficio depende positivamente del margen de la productivi-

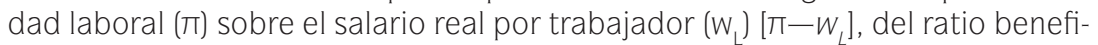
cio-salarios $(e=B M)$ y de la participación del beneficio en el producto $(\beta=B / Y)$; y negativamente del ratio capital-trabajo $\left(\theta\right.$, a precios constantes $\left.\left[{ }^{*}\right]\right)$, capitalproducto $(\delta)$ (inversa de la productividad del capital, $\Pi_{k}$ ) y capital-salarios $(\tau)$, considerando los deflactores de precios del producto $\left(P_{y}\right)$, el capital $\left(P_{k}\right)$ y el consumo $\left(\mathrm{P}_{\mathrm{c}}\right)$. Estos ratios asociados a la tecnología de la producción $-\mathrm{o}$ dimensiones de la composición del capital -, que analizaremos empíricamente en la siguiente sección, se expresan de la siguiente forma.

$$
\begin{aligned}
\text { Ratio capital-trabajo: } \theta & =\frac{K^{*}}{L} \frac{1}{P_{k}} \\
\text { Ratio capital-salarios: } \tau & =\frac{K}{W}=\theta \frac{P_{k}}{w_{L}} \\
\text { Ratio capital-producto: } \delta & =\frac{\theta}{\pi} P_{k y}
\end{aligned}
$$

Nótese que el segundo ratio $(\tau)$ depende también de la esfera de la distribuciōn del ingreso, al incorporar el salario, de ahí que en este trabajo nos centremos en los ratios $\theta$ y $\delta$. El primero posee cierta prioridad analítica debido a que pone en relación a los dos factores en los que se materializa la inversión, el capital fijo y la fuerza de trabajo (L), si bien esta medida no está exenta de controversias. ${ }^{6}$ Este ratio representa el nivel de mecanización del proceso económico - volumen de activos de capital por unidad de trabajo-, y constituye el primer fundamento para la mejora de la productividad laboral $(\pi)$, que se estima como el total del producto a precios constantes $\left(\mathrm{Y}^{*}\right)$ por unidad de trabajo (L), , por lo que, grosso modo, $\{\theta \rightarrow \pi\}$. Por tanto, el incremento de los ratios del capital $(\theta, \tau, \delta)$ presiona a la baja la rentabilidad, y por extensión, al crecimiento económico, pero al mismo tiempo permiten elevar la productividad. Y si ésta efectivamente se incrementa, descienden los costes de producción, lo que incide en los precios relativos, por lo que se destaca la relaciōn entre $P_{y}$ y $P_{k}$.

En consecuencia, estos dos factores, a saber: i) la eficiencia productiva del capital, o medida en la cual el stock de capital permite mejorar la productividad laboral $(\theta \rightarrow \pi)$; y ii) el índice de precios relativos $\left(P_{y}, P_{k}\right)$, o medida en la cual se pueda abaratar relativamente el precio de aquellos activos que permiten mejorar la productividad, determinarān el perfil del ratio capitalproducto - o alternativamente, su inversa, la denominada "productividad del capital".

$$
\Pi_{k}=\frac{\pi}{\theta} P_{y k}
$$

6 Efectivamente, en cuanto que relaciona categorias tan heterogéneas entre ellas, y por las particularidades de la metodología en el cálculo del capital (vêase Shaikh, 2016). 
Así pues, la relación entre la acumulaciōn de capital y la productividad es relevante por cuanto constituye un determinante fundamental del crecimiento económico, al incidir sobre la estructura de costes (competitividad).

Por otra parte, en el artículo se aludirá a dos versiones del producto: i) el PIB, según aparece en la contabilidad nacional, y ii) lo que se denominará como "valor nuevo" o "producto" (Y), a partir de deducir del PIB los sectores financiero e inmobiliario, junto a los servicios sociales y administraciones públicas. Esta medida será utilizada en los ratios del capital y la productividad laboral, y su metodología se explica por la consideración del trabajo improductivo (asalariado y mercantil) y por el propósito de eliminar cualquier sesgo procedente de una expansión relativamente elevada de las finanzas, lo inmobiliario o actividades no mercantiles. ${ }^{7}$

\subsection{ACUMULACIŌN Y DESARROLLO DESIGUAL: COMPARATIVA CENTRO-PERIFERIA}

Al comparar los procesos de acumulación de capital entre países, sobre todo con desigual nivel de desarrollo productivo, las categorías deben medirse en una misma moneda, en este caso una divisa internacional (dólares, \$), propia de las áreas centrales. Por ello, en primer lugar se abordan las asimetrías propias de las variables monetarias, pues entendemos que existe una integración orgánica entre lo real - productivo de valor - y lo monetario. En esta sección, tomamos como ejemplo una economía desarrollada (A) y una periférica, subdesarrollada o en vías de desarrollo (B), lo que supone una necesaria simplificaciōn metodológica para revelar las tendencias que cabe esperar que operen sobre estas últimas. Posteriormente, se abordan las tendencias propias de los ratios del capital y la productividad.

\subsubsection{ESFERA MONETARIA}

La relación entre el valor de las monedas, o tipo de cambio (TC), es fundamental en el análisis comparativo de las dinámicas productivas, ya que el primer condicionante de las paridades cambiarias es el nivel competitivo de los capitales (Astarita, 2010, Shaikh, 2016). Asī, su centro de gravedad -o nivel sobre el que tienden a fluctuar en el largo plazo - entre dos países son los costes laborales unitarios reales (Martínez-Hernández, 2017). Por tanto, el desarrollo tecnológico de una economía conduce o presiona -ejerciendo de tendencia- a la apreciación de su moneda, y lo contrario ocurre en una economía que pierde competitividad, lo que implica que en una economía atrasada su TC puede ubicarse por encima del correspondiente a la paridad del poder adquisitivo (PPA) (Astarita, 2010), dadas las disparidades entre los diversos índices de precios. 
Una primera aclaraciōn es necesaria: el análisis comparativo privilegia la utilizaciōn de los tipos de cambio de mercado en lugar de la PPA, como de hecho utilizaban los organismos internacionales hasta principios de 1990s. Ello se debe a que la perspectiva adoptada enfatiza el desarrollo productivo comparado de las economías, no el poder adquisitivo de sus habitantes (Freeman 2004, Saad-Filho 2014, Mateo, 2020).8 A los efectos del propósito de investigación, la adquisición de los elementos que permiten la mejora productiva, los bienes de capital, se lleva a cabo en divisa internacional a tipo de cambio de mercado, e incluso las deudas asociadas se vinculan a la misma paridad.

En principio, cabe esperar que las economías más competitivas tengan mayor estabilidad monetaria, reflejada en menor inflaciōn promedio. Asimismo, existe una presión estructural hacia el abaratamiento relativo del stock de capital, es decir, cierto aumento de $P_{y k}$, o al menos sincronía entre $P_{y}$ y $P_{k}$, lo cual se explica por el papel central que el stock de capital posee en el desarrollo productivo, rubro en el que las economías periféricas son menos competitivas y deben importar una parte considerable de los activos con mayor contenido tecnológico (Mateo, 2020).

Esta tendencia sería coherente con la disparidad de las medidas a PPA y a TC de mercado entre países desarrollados y periféricos. El primer índice se refiere al coste de la cesta de consumo, que es sustancialmente inferior en las áreas no desarrolladas, mientras que existe un único mercado internacional para los activos del capital. Es decir, existe un sistema dual de precios mundiales, con un coste global del capital y uno de carácter local para la fuerza de trabajo, dada la diversidad de mercados laborales en los que se establecen los salarios (Freeman, 2004). Por tanto, cabe esperar que la esfera monetaria amplifique las tendencias divergentes que, no obstante, brotan de la heterogénea capacidad productiva.

\subsubsection{CAPITAL Y PRODUCTIVIDAD}

Considerando la centralidad del capital para diferenciar el nivel de desarrollo de las economías, se deben comparar los diversos ratios del capital. En primer lugar, cabe esperar que el grado de mecanización sea superior en el país desarrollado, pero también puede disponer de mecanismos para evitar su excesivo incremento. Por ello, se pueden verificar las diferencias relativas para la economía central y periférica en cuanto a los medios (la mecanizaciōn) y los resultados (productividad): la productividad relativa de 'b' respecto de 'a' es el ratio de productividades en dólares, $\left(\pi_{b a}\right)^{\$}=\left(\pi_{b} / \pi_{a}\right)^{\$}$, lo que se debe comparar con el ratio capital-trabajo relativo $\left(\theta_{b a}\right)^{\$}=\left(\theta_{b} / \theta_{a}\right)^{\$}$, por lo que

8 Nótese que un dólar a PPA es una unidad de cuenta ficticia, que no existe en ningún sentido fundamental, ni para realizar pagos ni para contabilizar transacciones. Aunque ello no implica en absoluto que carezca de pertinencia analítica, no es relevante para el análisis de la acumulación de capital, la capacidad productiva y la fortaleza económica que, de manera tendencial (o subyacente), explican el desarrollo productivo y el poder geopolítico. 


$$
\sigma_{b a}=\left(\frac{\theta}{\pi}\right)_{b a}^{\$}
$$

Si la distancia de ratios $\theta$ es inferior al diferencial de $\pi$, entonces cabe afirmar que el stock de capital es relativamente ineficiente en la economía más atrasa$\mathrm{da}, \mathrm{y} \sigma_{\mathrm{ba}}>1$, o bien, que el nivel de mecanización sería excesivamente elevado de acuerdo al nivel de productividad que se logra. En este caso, nótese que esta ineficiencia puede acompañar a un encarecimiento relativo del capital $\left(P_{k y}\right)$, y se ampliaría la diferencia en los indices de las productividades relativas del capital. Efectivamente, las economías periféricas pueden adolecer de todavía una mayor desconexión entre los ratios del capital, ya que $\delta=(\theta \pi) P_{k y}$, y se ve así doblemente impulsado al alza, dado el aumento de $\theta$ (y menor incremento de $\pi$ ) junto al de $P_{k y}$. Como se hizo en la expresión (6), se puede hacer el mismo ejercicio con el ratio capital-producto (productividad del capital), relación que se denotaría por $\mu$, y comparar $\sigma$, para ilustrar la relación relativa de los ratios de capital respecto de la divergencia en la productividad laboral con la economía de referencia.

$$
\mu_{b a}=\left(\frac{\delta}{\pi}\right)_{b a}^{\$}
$$

La hipótesis de partida es, por una parte, que los ratios del capital estān asociados a la productividad laboral, relación general que no obstante se ve mediada por aspectos concretos del contexto, así como la estructura de la inversión (capital) por activos y sectores económicos. Por otra, se considera que existe una serie de obstáculos estructurales a la convergencia y al equilibrio, es decir, afirmo que la dinámica de acumulaciōn se caracteriza por un desarrollo desigual, ${ }^{9}$ con factores estructurales que tienden a reproducir, y tal vez a ampliar, las divergencias productivas internacionales. En definitiva, se plantea que el subdesarrollo productivo puede manifestarse en un nivel relativamente elevado de sus ratios de capital respecto de su nivel de productividad. Considerando una comparación en divisa internacional, constituye un obstáculo para su proceso de acumulaciōn, que contribuiría a reproducir las diferencias centro-periferia.

\section{Resultados EMPÍRICOS}

\section{1. EVOLUCIŌN DEL CAPITAL Y LA PRODUCTIVIDAD}

La inversiōn bruta (FBCF) respecto del PIB es inferior en Brasil, oscilando apenas entre el $16 \%$ y el 19\%, es ligeramente superior en EEUU, 20-22\% hasta 2008, y posteriormente 18-19\%, mientras que en España pasa del 22\%

9 El término desarrollo desigual implica que no existe una tendencia hacia la convergencia en la economía mundial, y que el proceso de acumulaciōn y desarrollo no es uniforme por sectores y áreas geográficas. No obstante, no excluye la posibilidad de que existan dinámicas convergentes. 
en 1995 al $31 \%$ en 2006-07, pero descendiendo después incluso por debajo del 20\% en 2012 (tabla 1). Si se deduce la inversión residencial, el monto relativo de inversión es no obstante muy similar en España y EEUU, y la diferencia también se estrecha con Brasil, donde este rubro de la FBCF es sensiblemente inferior a EEUU.

A precios constantes, como muestra la gráfica 1, el perfil de la inversión es diferente en EEUU y España respecto de Brasil. En las primeras, se aprecia un ciclo expansivo hasta 2006-07, con un retroceso posterior, aunque en la primera se recupera a partir de 2009. Por su parte, en Brasil existe un estancamiento inicial que sólo es superado a partir de la fase alcista iniciada en 2003. Globalmente, el volumen de inversión total crece en mayor medida en Brasil —casi un 85\% hasta 2013-, por 56,8\% en EEUU y 34,4\% en España, si bien sólo supera a estas dos últimas en virtud del retroceso que experimentan en la Gran Recesión. Esta dinámica en los flujos de inversión se ha traducido en un incremento acumulado del PIB superior en Brasil (81\%), seguido de EEUU (57\%), y finalmente España (48\%), ${ }^{10}$ mientras que el producto $\left(\mathrm{Y}^{*}\right)$ aumenta algo menos que el PIB en Brasil (65\%), igual en EEUU (57\%), y todavía menos en España (31\%).

GRÁFICA 1. DINÁMICA DE LA INVERSIÓN BRUTA TOTAL.

SERIES A PRECIOS CONSTANTES EN MONEDA NACIONAL, $1995=100$

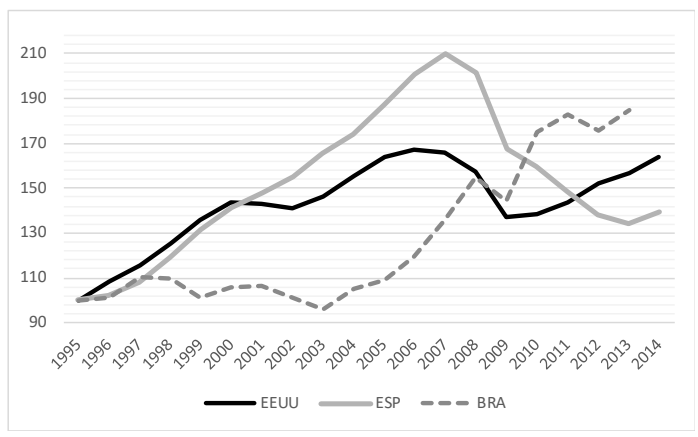

Fuente: BEA (2017), FBBVA (2017), IPEA (2017).

Los flujos de inversión se materializan en un determinado stock de capital (K). A lo largo del período 1995-2014, el volumen del stock de capital neto no residencial a precios constantes $\left(K^{*}\right)$ crece más intensamente en España, multiplicándose por 2,35, mientras que en EEUU lo hace por 1,56 y en Brasil se multiplica por 1,43, y ello aunque - como se explicó anteriormente- el volumen de FBCF crece globalmente más en Brasil, seguido por EEUU y España. ${ }^{11}$

10 Si se toma el crecimiento del PIB pc, los resultados agregados son bastante más similares, ya que el crecimiento acumulado es del 36,6\% en Brasil, 31,1\% en EEUU y 24\% en España (FMI, 2017a).

11 Esta discrepancia entre los flujos de la FBCF y la evolución del capital en términos netos explica la diferente relación entre el stock de capital y el producto en las tres economías, como se apreciará en la tabla 2. 
En el siguiente grupo de ilustraciones (gráfica 2) se puede visualizar la dinámica de los determinantes del ratio capital-producto (inversa de la productividad del capital), a saber: el ratio capital-trabajo, la productividad laboral y la relación de precios. Este período se caracteriza por un magro incremento de $\theta$, ya que sólo en España crece por encima del 2\% anual - promedio del $2.57 \%$-, mientras que en EEUU aumenta al 1,17\% por año, y en el caso de Brasil, apenas el 0,75\%.

En términos globales, existe una estrecha relación entre el ratio $\theta$ y la productividad en EEUU y Brasil, junto a España hasta 2008, momento a partir de cual se produce una destacada desconexiōn. ${ }^{12}$ La relación entre ambas categorías - 0 eficiencia productiva de $\mathrm{K}^{*} / \mathrm{L}-$, se muestra en la gráfica inferiorderecha. Claramente, el caso español resulta absolutamente atípico, ya que este indice de eficiencia desciende un 32,6\%, mientras que en EEUU aumenta un $4,8 \%$, y en Brasil permanece constante $(0,58 \%)$, con un aumento productivo del $24 \%$ entre 1999 y 2008, momento a partir del cual desciende.

Gráfica 2. Determinantes de la productividad del CAPITAL Y eficiencia productiva del índice de MECANIZACIŌN $(1995=100)$
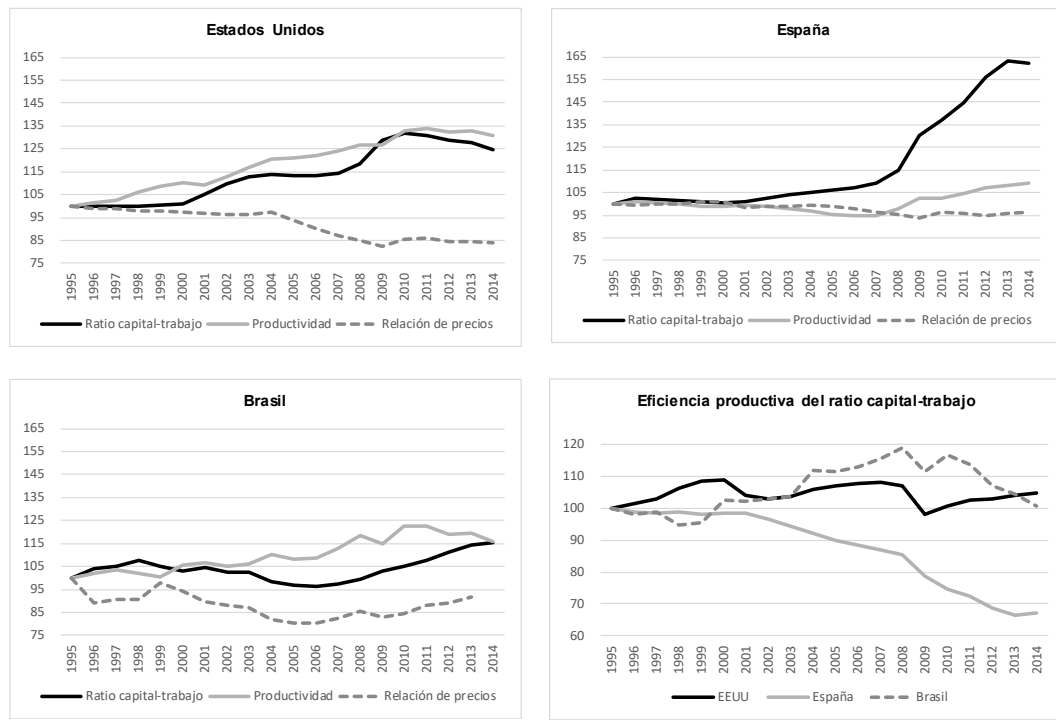

Nota. Series del ratio capital-trabajo (precios constantes, $\theta$ ), productividad laboral y ratio de precios $\left(P_{y k}\right)$, y eficiencia productiva del ratio capital-trabajo (productividad/ $\theta$ ).

Fuente: BEA (2017), FBBVA (2017), IBGE (2017), INE (2017), IPEA (2017), Marquetti y Porsse (2014), Morandi (2015).

$12 \mathrm{O}$ lo que es lo mismo, entre $\mathrm{K}^{*}$ y el producto $\mathrm{Y}^{*}$, ya que el denominador es el mismo (L) lo cual se deriva de la relaciōn entre la inversiōn y el producto. 
GRĀFICA 3. EVoluCIŌN COMPARADA dE LA PRODUCTIVIDAD DEL CAPITAL $(1995=100)$

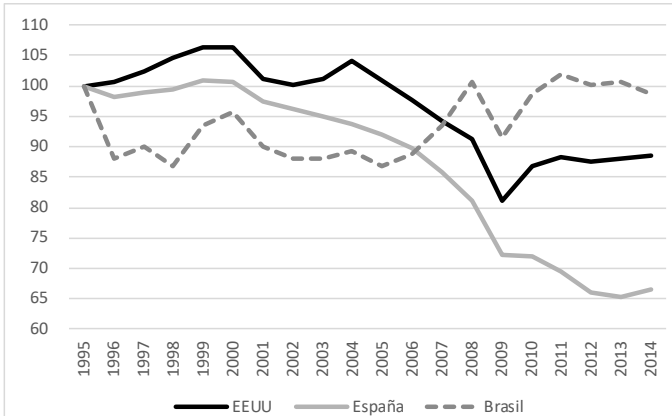

Fuente: BEA (2017), FBBVA (2017), IBGE (2017), INE (2017), IPEA (2017), Morandi (2015).

En los tres casos, hay que destacar que el stock de capital se encarece relativamente respecto del producto (descenso del ratio $P_{y k}$ ), y paradójicamente, es en EEUU donde el índice $P_{y k}$ más cae $(15,6 \%)$, seguido de Brasil $(8,2 \%)$ y España (apenas 4,5\%). Ahora bien, en el primer caso, el descenso se concentra entre 2004 y 2007-09, cuando disminuye un $11 \%$ (15\% hasta 2009), en España en 2004-09 cae un 5,5\%, y en Brasil se produce una caída del 17\% entre 1999 y 2005. En este último, el ratio cae un $11 \%$ en 1996, por lo que si se cuenta desde este año (1996-14), el ratio hubiera permanecido constante hasta 2012. Así pues, se ha de destacar esta particularidad: se erosionan los efectos de la deslocalizaciōn productiva en EEUU (Beitel, 2009), España no se beneficia de un euro apreciado para abaratar los activos del capital, y en Brasil se revierte una de las tendencias propias de la etapa de la industrialización por sustituciōn de importaciones (véase Mateo, 2018).

A partir del análisis de las categorías $\theta, \pi$ y $P_{y k}$, resulta que $\delta(K / Y)$ se incrementa hasta un 50\% en España, frente a un 13\% en EEUU y únicamente un 1,4\% en Brasil. O inversamente, como aparece en la gráfica 4, la economía brasileña ha logrado que su productividad del capital $\left(\Pi_{k}\right)$ se mantenga constante, ya que el aumento de $\mathrm{K}$ se traslade en un 98,6\% al producto $(\mathrm{Y})$, superando a EEUU, donde ha caído un 11,6\%, mientras que España queda lejos, con una caída del 33,5\%. ${ }^{13}$

Estos resultados agregados se complementan en la tabla 1 considerando las etapas de crecimiento y recesión. Estos datos destacan la profundidad de

13 En el caso de España se debe tener en cuenta la burbuja inmobiliaria, ya que los activos relacionados con la construcciōn y los sectores con menor composiciōn de capital, y por tanto menos productivos y más inflacionarios, han sido los más dinámicos del proceso de acumulaciōn. Sobre este tema, remito a Mateo (2017) y Mateo y Otro (2018). Respecto de EEUU, precisamente el período posterior a 1995 rompe una tendencia alcista en $\prod_{k}$ desde 1982 (Beitel, 2009), mientras que en Brasil esta dinámica invierte la extraordinaria caída de las décadas anteriores (Mateo, 2018b), lo que puede relacionarse con la reestructuración sectorial (quiebras de actividades manufactureras menos productivas con la liberalización económica) y la estabilización cambiaria. 
las transformaciones acontecidas en este período, tanto por la erosión de la superioridad de la economía de EEUU, habida cuenta de la dinámica de las categorías de la acumulación, como por las diversas combinaciones de tendencias en las fases de crecimiento y crisis.

TABLA 1. TASAS de VARIACIŌN ANUAL PROMEDIO dE LAS CATEGorías dE LA ACUMULACIŌN DE CAPITAL

\begin{tabular}{|c|c|c|c|c|c|c|c|c|c|c|}
\hline \multicolumn{11}{|c|}{ Fases expansivas } \\
\hline & Período & PIB & $Y^{*}$ & $\mathbf{K}^{*}$ & $\mathbf{L}$ & $K^{*} / L$ & $\mathbf{P}_{\mathrm{yk}}$ & $\pi$ & $\Pi_{k}$ & $\Pi_{k}^{*}$ \\
\hline \multirow{3}{*}{ EEUU } & $1995-00$ & 4,30 & 4,63 & 2,87 & 2,64 & 0,22 & $-0,52$ & 1,94 & 1,26 & 1,72 \\
\hline & $2003-07$ & 2,87 & 3,05 & 2,03 & 1,16 & 0,86 & $-2,07$ & 1,88 & $-1,20$ & 1,00 \\
\hline & $2010-14$ & 2,08 & 2,51 & 1,17 & 1,83 & $-0,65$ & 0,42 & 0,66 & 1,74 & 1,32 \\
\hline \multirow{2}{*}{ ESP } & $1995-00$ & 4,09 & 3,89 & 4,20 & 4,10 & 0,10 & 0,16 & $-0,20$ & 0,12 & $-0,30$ \\
\hline & $2000-07$ & 3,56 & 2,62 & 4,45 & 3,25 & 1,16 & $-0,66$ & $-0,61$ & $-2,23$ & $-1,75$ \\
\hline \multirow{4}{*}{ BRA } & $1995-97$ & 2,76 & 1,69 & 2,32 & $-0,11$ & 2,43 & $-4,74$ & 1,80 & $-5,08$ & $-0,61$ \\
\hline & 1999-03 & 2,35 & 3,75 & 1,64 & 2,39 & $-0,73$ & $-2,84$ & 1,33 & $-1,49$ & 2,07 \\
\hline & $2004-08$ & 4,81 & 4,91 & 2,09 & 2,66 & $-0,55$ & $-0,32$ & 2,19 & 2,71 & 2,76 \\
\hline & 2010-14 & 4,39 & 1,88 & 3,98 & 1,67 & 2,27 & 2,64 & 0,21 & 1,51 & $-2,01$ \\
\hline \multicolumn{11}{|c|}{ Fases recesivas } \\
\hline \multirow{2}{*}{ EEUU } & 2001 & 0,98 & $-1,26$ & 3,43 & $-0,42$ & 3,87 & $-0,67$ & $-0,84$ & $-4,94$ & $-4,53$ \\
\hline & 2008-09 & $-1,54$ & $-2,61$ & 2,27 & $-3,62$ & 6,11 & $-2,64$ & 1,05 & $-7,21$ & $-4,76$ \\
\hline ESP & $2009-13$ & $-1,07$ & $-1,97$ & 2,54 & $-4,12$ & 6,94 & $-0,12$ & 2,24 & $-4,44$ & $-4,40$ \\
\hline \multirow{2}{*}{ BRA } & 1998-99 & 0,14 & 0,81 & 2,48 & 2,29 & 0,18 & 3,71 & $-1,44$ & 1,83 & $-1,62$ \\
\hline & 2009 & $-0,33$ & $-2,35$ & 4,29 & 0,70 & 3,56 & $-3,37$ & $-3,03$ & $-9,04$ & $-6,36$ \\
\hline
\end{tabular}

Nota. Esta periodización se ha establecido en función de la evolución del PIB, y se muestra en moneda nacional, siendo * a precios constantes.

Fuente: BEA (2017), FBBVA (2017), IBGE (2017), INE (2017), IPEA (2017), Marquetti y Porsse (2014), Morandi (2015)

En términos de $\prod_{k}$ a precios constantes, el volumen de capital es sustancialmente productivo en EEUU en las tres fases expansivas, en Brasil de manera muy destacada en las dos fases intermedias (entre 1999 y 2008), pero en España la masa de capital origina siempre un crecimiento menor del producto. Se aprecia a su vez una ausencia de conexión entre el ratio capital-trabajo $(\theta)$ y la productividad laboral, ya que existen todo tipo de combinaciones, lo que requeriría información fidedigna sobre la tasa de utilización de la capacidad instalada.

La cantidad de empleo utilizado muestra por su parte una gran variabilidad, sobre todo en el caso de Brasil, lo que sugiere la existencia de cambios en la estructura económica, ${ }^{14}$ mientras que España ha tenido un periodo de crecimiento de carácter trabajo-intensivo, aunque descendente en la segunda parte, pero superior en cualquier caso al 3\% anual.

14 Asî, con tasas de acumulación del 2,1-2,3\% anual el empleo llega a descender al 0,1\% y a aumentar al 2,6\%, mientras que en la última fase una aceleraciōn del ritmo de crecimiento del stock de capital sólo impulsa el empleo un 1,6\% anual. 
En definitiva, no se verifica un patrōn que distinga a estas economías en cuanto a las fases de acumulación y crisis, salvo la mayor estabilidad relativa de la economía de EEUU.

\subsection{Cuestiones MonetARIAS}

Como cabía esperar, la inflaciōn es sustancialmente superior en Brasil respecto de EEUU y España, a pesar de que este período se caracteriza por lograr una importante estabilidad monetaria en el paîs sudamericano. ${ }^{15}$ En EEUU, $P_{y}$ se incrementa un 44,5\% en estas dos décadas, en España lo hace sólo 10 puntos más (55,1\%), si bien se ha de tener en cuenta que lo limitado de este diferencial se explica por la Gran Recesión, cuando su deflactor de precios se mantiene prácticamente estancado, 0,11\% anual. En cuanto a Brasil, el contraste es profundo, ya que el deflactor de precios crece un 352\% acumulado, con 3 años en los cuales supera el 10\%, incluido 1995, con un 93\% de inflaciōn.

Para España y Brasil, este período se caracteriza asimismo por una dinámica del tipo de cambio en el cual el diferencial respecto del dōlar es inferior a la inflación (gráficas 4 y 5). En España, la paridad nominal se deprecia casi un 50\% hasta 2001, para apreciarse posteriormente hasta el estallido de la crisis, por lo que en el conjunto del período, la relación nominal con el dólar permanece constante. La evoluciōn del TC en Brasil se caracteriza igualmente por una depreciación, más intensa en este caso, hasta 2003. En este año, el tipo de cambio del Real es 3,3 veces el vigente en 1995, pero con el ciclo ascendente posterior, el Real se aprecia notablemente, sobre todo hasta 2011 , para volver a depreciarse justo después, y en 2014 la paridad nominal es 2,5 veces superior a 1995. Por tanto, lo específico de este período es el sexenio anterior al estallido de la crisis, como se aprecia en la gráfica 5.

Respecto del nivel correspondiente a la PPA, tanto para España como para Brasil el tipo de cambio se aprecia relativamente, lo que constituye uno de los rasgos más destacados de este período. En el primero, se produce una depreciaciōn relativa del 38\% hasta 2001, que se corrige después, mientras que en el segundo la oscilación es más intensa. Hasta 2002 el ratio (TC nominal/ PPA) se multiplica por 2 -índice de 202,38, con 1995 = 100-, pero la caída posterior -apreciación relativa - lleva a que en 2011 el índice sea un 32\% inferior respecto de 1995. Finalmente, la apreciación relativa alcanza un 18\% en Brasil en 2014, por 6,3\% en España respecto del nivel inicial.

15 En los años anteriores la inflaciōn había sido del 400\% y 970\% en 1991-92, para llegar posteriormente a casi el $2000 \%$ en 1993 y pasar del $2200 \%$ en 1994 , por lo que el $93 \%$ registrado en 1995 supuso un destacado logro del Plan Real (FMI, 2017a). 
Gráfica 4. Dināmica comparada del TIPO de CAMBIO NOMINAL CON EL DÓLAR Y LA INFLACIŌN EN ESPAÑA Y BRASIL $(1995=100)$

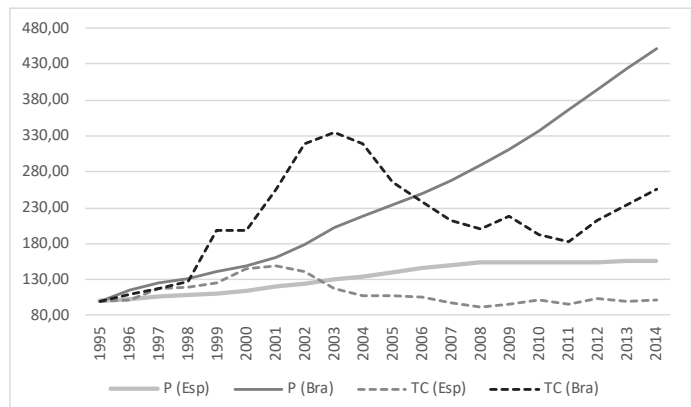

Nota. Inflaciôn (deflactor del PIB) (P) y tipo de cambio (moneda nacional/dólar, TC). Fuente. FMI (2017a, 2017b), OCDE (2017).

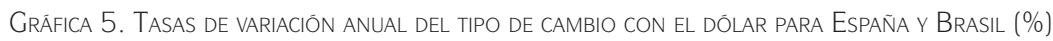

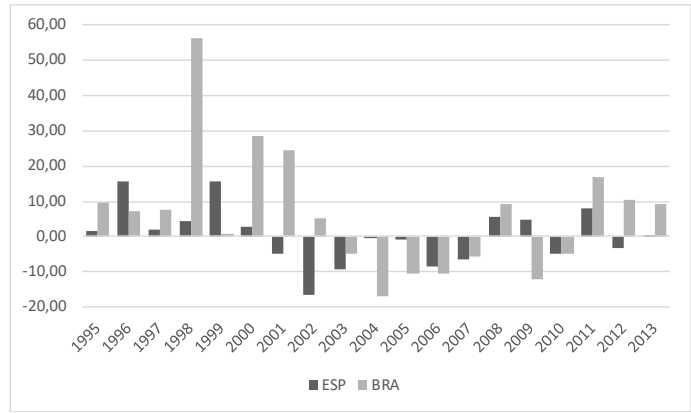

Nota. Moneda doméstica por unidad de dôlar. Fuente: FMI (2017b), OCDE (2017).

GRÁFICA 6. VARIACIŌN ACUMULADA DE LA INFLACIŌN, EL TIPO DE CAMBIO Y EL RATIO TIPO DE CAMBIO-COEFICIENTE DE LA PPA, EN 1995-2014 (1995= 100)

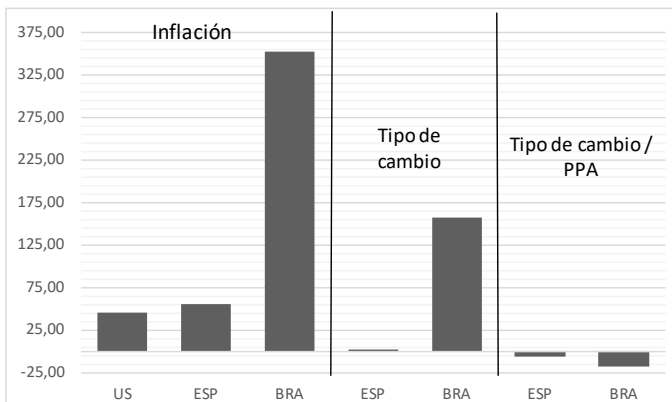

Fuente: FMI (2017a, 2017b), OCDE (2017). 
Así pues, a pesar del éxito de los programas de estabilizaciōn, la inflación es más volátil y relativamente más intensa en la economía semiperiférica, con un amplio diferencial en el aumento de precios entre las dos economías desarrolladas y Brasil. A su vez, el tipo de cambio es estable entre EEUU y España, mientras que el Real brasileño adolece de mayores oscilaciones y una depreciación neta que le lleva a triplicarse en 2002-04, y acabar siendo más del doble en 2012-14, aunque las paridades de España y Brasil se aprecian respecto del nivel de la PPA, a pesar de la presión estructural antes expuesta. Por tanto, la capacidad productiva de Brasil debería avanzar más de 2,5 veces más rápido que en España y EEUU para que exista convergencia en términos de la productividad laboral, lo que se aborda a continuaciōn.

\subsection{Comparativa de niveles absolutos}

Esta segunda parte del análisis comparativo se centra en la divergencia de niveles absolutos de diversas categorías propias del proceso de acumulación de capital, tomando Estados Unidos como referencia (EEUU = 100). En primer lugar, el ratio capital-trabajo en dólares a precios constantes $\left(\mathrm{K}^{*} / \mathrm{L}\right)^{\S}$ oscila en España entre 32 y 63\% del correspondiente a EEUU, aunque este máximo es un producto de la Gran Recesión y del aumento del desempleo - caída del denominador (L) - , ya que hasta 2010 era del 52\%. Brasil tiene un nivel de mecanización relativamente muy bajo, y con una tendencia descendente en la segunda mitad de los noventa, cuando cae del 25 al 13\% para oscilar posteriormente, en los 2000s, entre 7 y $13 \%$.

El nivel relativo de los ratios $\delta(K / Y)$, sin embargo, es sustancialmente diferente. Si bien en EEUU es superior a España y Brasil, las diferencias son inferiores, y además, no guarda relación con los niveles de productividad. Entre 1995 y 2003, el ratio de España representaba en torno al 68-72\% del correspondiente a EEUU, y sólo en la fase de la larga crisis este ratio se incrementa relativamente hasta un 90-92\% en 2012-14. Por el contrario, en Brasil es muy diferente, ya que entre 1996 y 2005 este ratio está muy cerca de EEUU (90-98\%), pero a partir de ese año se produce una caída hasta oscilar entre el 70 y $73 \%$. Así pues, como se esperaba, se aprecia una profunda divergencia entre los ratios capital-trabajo y capital-producto tanto en España como en Brasil - principalmente- respecto de EEUU.

A partir de esta evolución comparada, pasamos a abordar los índices de la productividad laboral. En la tabla 2 se muestran estimaciones de la misma a partir del PIB total y la parte que consideramos productivo (Y), por empleado y hora de trabajo, y utilizando los TC de mercado y a PPA. Metodológicamente, es preciso destacar la disparidad de cifras que muestran las series de The Conference Board (TCB), CEPAL, OCDE, Banco Mundial y las Penn World Tables (PWT) [Feenstra y otros, 2017], lo que resulta inquietante por su amplitud, y 
justifica este ejercicio comparativo. ${ }^{16}$ Las diferencias entre los índices de productividad del total de la economía y el ámbito productivo no son relevantes. En el caso de España, al principio del período este último era 2-3 puntos superior relativamente, pero a partir de 2005 desaparece el diferencial. No obstante, ello supone que la divergencia del ámbito productivo se amplía en mayor medida que para el total de la economía.

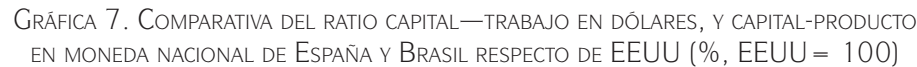

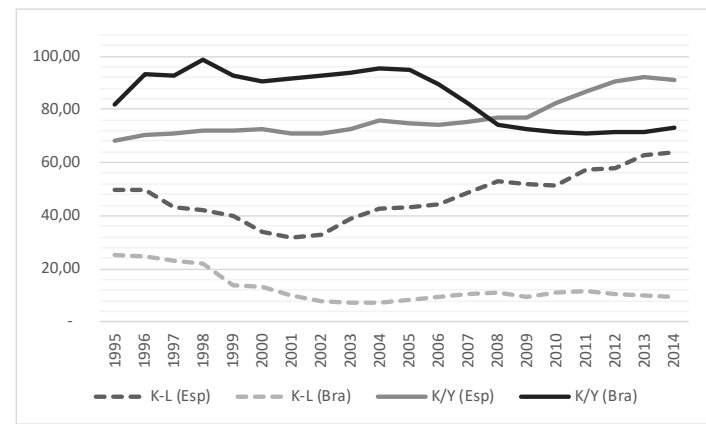

Fuente. BEA (2017), IBGE (2017), INE (2017), IPEA (2017), Marquetti y Porsse (2014), Morandi (2015).

Hay dos aspectos cruciales en esta ilustración. En primer lugar, el contraste entre los niveles de productividad a tipo de cambio de mercado y a PPA. Como cabía esperar, el segundo procedimiento reduce sustancialmente la divergencia de los valores absolutos, o más correctamente, su utilización por parte de los organismos internacionales oculta la magnitud de la disparidad entre los países. Para España, la diferencia oscila entre los 12 y los 40 puntos porcentuales - si se compara con la serie del Banco Mundial-, con una tendencia descendente de esta diferencia. En cuanto a Brasil, desde 1999 la diferencia con EEUU resulta superior cuando se relaciona con el nivel relativo de su productividad. Asī, su PIB por trabajador oscila entre el 9 y el $16 \%$ de EEUU, pero en el resto de organismos a PPA llega al 24-27\%, por lo que este nivel puede llegar a ser más del doble a PPA. ${ }^{17}$

16 En el caso de España, las discrepancias ocurren sobre todo en los primeros años de la serie, antes de la adopción del Euro: en 1995-97, su nivel relativo de productividad era del 92-95\% según el Banco Mundial, y sólo del 75-77\% para la OCDE. Para el caso de Brasil, estas diferencias son infimas con estos organismos, pero sí es significativa la comparaciōn cuando se utiliza la serie del PIB de la CEPAL, que rebaja su nivel de productividad unos 5-7 puntos, hasta el 23\% en los primeros años. 17 La serie de Brasil con el PIB de CEPAL tiene valores anómalos, ya que son inferiores a los correspondientes a los organismos internacionales, pero en cualquier caso entre 6 y 12 puntos porcentuales superiores a los datos aqui calculados, a partir de 1999. Además, la última serie de TCB con precios de 2016 eleva unos 2-3 puntos el nivel relativo de productividad de Brasil, manteniendo la evolución. 


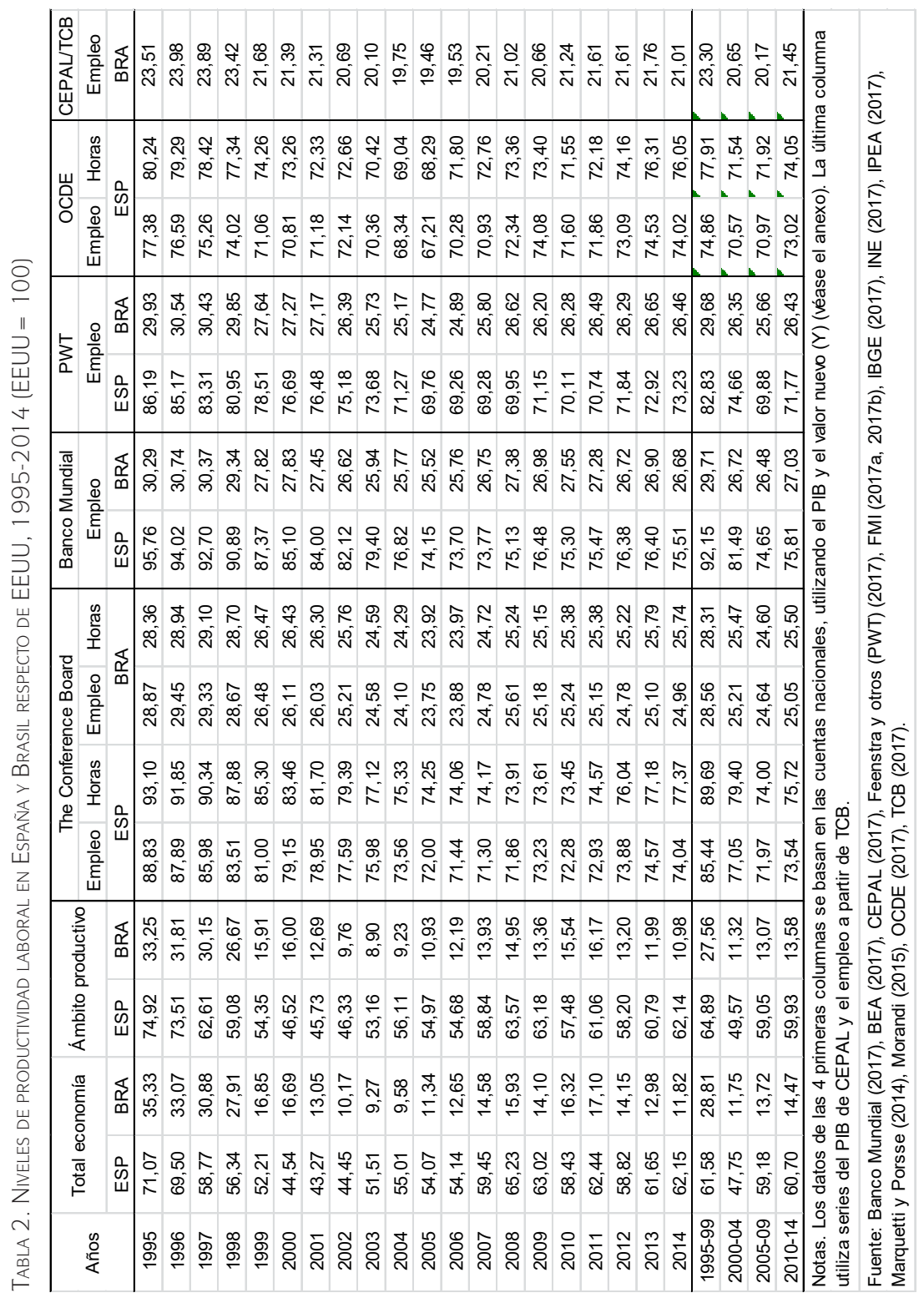


En segundo lugar, estas series no permiten afirmar la existencia de ninguna tendencia hacia la convergencia entre España y Brasil respecto de EEUU. Muy al contrario, todos los índices de productividad muestran una ampliación de la divergencia. Ello se comprueba con los promedios quinquenales incluidos en la parte inferior de la tabla, que eliminan el sesgo de la elección del primer y último año de la serie. Aunque las variaciones dependen de la fuente, el principal contraste se produce, nuevamente, entre los datos calculados a TC de mercado y a PPA.

A partir de estos datos, se constata que el ratio capital-trabajo en dólares constituye una adecuada aproximaciōn a los diferenciales de productividad, mientras que el ratio capital-producto no tiene el mismo poder explicativo, ya que la dependencia de las importaciones de los elementos del stock de capital incide tanto en el numerador como en el denominador. De ahí la ausencia de un patrón claro en su evolución comparada. Este desajuste, o perfil contrapuesto, se aprecia en la tabla 3, donde se muestran los coeficientes $\sigma$ y $\mu$ antes expuestos.

TABla 3. Ratios de los diferenciales entre los ratios del CAPITAL Y LA PRODUCTIVIDAD LABORAL RESPECTO DE EEUU (\%)

\begin{tabular}{|l|l|l|l|l|}
\hline \multirow{2}{*}{ Años } & \multicolumn{2}{|c|}{ Coeficiente $\sigma$} & \multicolumn{2}{c|}{ Coeficiente $\mu$} \\
\cline { 2 - 5 } & ESP & BRA & ESP & BRA \\
\hline 1995 & 0,66 & 0,75 & 0,91 & 2,45 \\
\hline 1996 & 0,68 & 0,78 & 0,95 & 2,93 \\
\hline 1997 & 0,69 & 0,77 & 1,13 & 3,07 \\
\hline 1998 & 0,71 & 0,83 & 1,22 & 3,69 \\
\hline 1999 & 0,73 & 0,87 & 1,33 & 5,84 \\
\hline 2000 & 0,73 & 0,84 & 1,56 & 5,67 \\
\hline 2001 & 0,70 & 0,81 & 1,55 & 7,23 \\
\hline 2002 & 0,71 & 0,79 & 1,53 & 9,49 \\
\hline 2003 & 0,73 & 0,80 & 1,37 & 10,53 \\
\hline 2004 & 0,76 & 0,78 & 1,36 & 10,31 \\
\hline 2005 & 0,79 & 0,78 & 1,36 & 8,66 \\
\hline 2006 & 0,81 & 0,78 & 1,36 & 7,36 \\
\hline 2007 & 0,82 & 0,76 & 1,27 & 5,91 \\
\hline 2008 & 0,83 & 0,74 & 1,21 & 4,95 \\
\hline 2009 & 0,82 & 0,72 & 1,22 & 5,41 \\
\hline 2010 & 0,89 & 0,70 & 1,43 & 4,61 \\
\hline 2011 & 0,94 & 0,73 & 1,42 & 4,37 \\
\hline 2012 & 0,99 & 0,80 & 1,55 & 5,40 \\
\hline 2013 & 1,03 & 0,82 & 1,51 & 5,95 \\
\hline 2014 & 1,03 & 0,85 & 1,46 & 6,66 \\
\hline
\end{tabular}

Fuente: BEA (2017), FBBVA (2017), FMI (2017a, 2017b), IBGE (2017), INE (2017), IPEA (2017), Marquetti y Porsse (2014), Morandi (2015), OCDE (2017) 
El ratio $\theta$ respecto de EEUU es en España y Brasil relativamente inferior a la productividad relativa. En España, esta dinámica comparada tiende no obstante a la convergencia, sobre todo a partir de 2011, y en Brasil oscila en torno al 70-87\%. Por ello, en estas economías el grado de mecanización relativo es inferior al nivel de productividad que poseen respecto de EEUU.

Por otra parte, el ratio capital-producto es relativamente superior en España y, sobre todo, en Brasil. En el primer caso, posee entre un 20 y un 50\% de exceso respecto del diferencial de productividad a partir del establecimiento de los tipos de cambio fijos en la Eurozona. En cuanto a Brasil, el ratio resulta muy superior, con un incremento hasta 2003, cuando posee un nivel 10 veces superior al que le correspondería de acuerdo a su productividad relativa con EEUU. Nótese que este movimiento se corresponde con la depreciación nominal del Real, como antes se expuso, y que alcanzó un 235\% en esos ocho primeros años de la serie. Posteriormente, el ratio K/Y se estabiliza en un exceso de 4 a 6 veces el diferencial en términos de productividad con EEUU. Así pues, aunque $\theta$ es relativamente bajo dado el diferencial de productividad, la disparidad se manifiesta en el alza del ratio capital-producto, excesivo en funciōn del diferencial de productividad con EEUU, sobre todo en el caso de Brasil.

\section{Conclusiones}

La FBCF respecto del PIB ha sido superior en España hasta el estallido de la Gran Recesiōn, pero debido al peso de la inversiōn residencial, mientras que en Brasil ni siquiera llega al 20\%. Sin embargo, el nivel de mecanización $\left(\mathrm{K}^{*} / \mathrm{L}\right)$ ha crecido relativamente poco en estas economías, pero en mayor medida en España, si bien es un resultado engañoso, ya que se explica por la reestructuración del empleo debido a la crisis, y posiblemente con una caída de la utilización de la capacidad instalada. Por ello, es en EEUU donde más ha avanzado la productividad, por encima del 30\% total, el doble que en Brasil y el triple que España.

Los ratios de precios $\left(\mathrm{P}_{\mathrm{yk}}\right)$ han tenido un comportamiento diferente al que caía esperar, fruto de la dinámica cambiaria: el capital se ha encarecido relativamente más en EEUU, por encima de Brasil - si bien hay que precisar que hasta el estallido de la Gran Recesión este encarecimiento relativo en Brasil fue superior - , y de España. Por ello, la productividad del capital tiene un mejor comportamiento en EEUU hasta la crisis mundial (2007), mientras que se mantiene constante en Brasil a pesar de las oscilaciones, y en el caso de España se produce un descenso desde 2000 que se acelera con la Gran Recesión, y lleva a un descenso total de casi el 35\%.

Los niveles relativos de $\theta\left(\mathrm{K}^{*} / \mathrm{L}\right)$ en dólares reproducen, y explican, las diferencias de productividad, pues en EEUU es mayor que en España, y en ésta superior a Brasil. El análisis ha revelado que: i) no existe un exceso en Brasil respecto de EEUU, pues el diferencial es superior al que tiene con EEUU en tér- 
minos de productividad. Por ello, $\sigma_{\text {Bra,EEUU }}<1$ en todo el período. Sin embargo, el ratio $\delta(K / Y)$ sí ha sido relativamente elevado, ya que $\mu_{\text {Bra,EEUu }}>1$, y es más, ha oscilado entre 4 y 10 desde 1999; ii) en España se produce una aguda caída de esta eficiencia relativa de $\theta$ en dólares, pues $\sigma_{\text {ESP,EEUU }}$ pasa de 0,6 a coincidir con el diferencial de productividad con EEUU, pero $\mu_{\text {ESP,EEUU }}>1$, si bien no alcanza los niveles de Brasil. Ello muestra el alcance que para la economía menos desarrollada tienen los factores que presionan al alza el ratio capital-producto.

A pesar de las particularidades del contexto (estabilidad monetaria, apreciaciōn cambiara en España y Brasil), y de su mayor nivel de crecimiento económico, estas dos décadas no muestran signo alguno de una tendencia hacia la convergencia en productividad con EEUU expresada en dólares. Muy al contrario, España pasa de estar a 25 puntos porcentuales (Y) (29 puntos en cuanto al PIB), a alejarse 28 en 2014, y la productividad en Brasil respecto de EEUU pasa de un tercio al 10-15\% a partir de 1999. En este sentido, se ha constatado el mayor diferencial en productividad a tipo de cambio de mercado en ambos casos, y relativamente superior en Brasil, respecto de los índices a PPA. En este último caso, la PPA también oculta el grado en el cual se amplía la divergencia -más de 20 puntos, en lugar de apenas 3 puntos porcentuales.

Finalmente, este estudio señala ciertas líneas de investigaciōn para profundizar: Ios resultados obtenidos revelan la complejidad de la relación entre la acumulación de capital y el crecimiento del producto, lo que amerita analizar la composición por activos y la estructura sectorial de la economía, si bien no existe disponibilidad de esta informaciōn para Brasil.

\section{REFERENCIAS BIBLIOGRÁFICAS}

Astarita, R. (2010): Economía política de la dependencia y el subdesarrollo, Universidad Nacional de Quilmes Ediciones, Buenos Aires.

Banco Mundial (2017): World Development indicators, Washington DC.

BEA (2017): National Income and Product Accounts, Bureau of Economic Analysis, US department of Commerce.

Beitel, K. (2009): "The Rate of Profit and the Problem of Stagnant Investment: a Structural Analysis of Barriers to Accumulation and the Spectre of Protracted Crisis", Historical Materialism, 17(4), 66-100.

Buendia, L. y Molero-Simarro, R. (2018): The Political Economy of Contemporary Spain. From Miracle to Mirage, Routledge, Londres.

Cámara, S. (2003). Tendencias de la rentabilidad y de la acumulación del capital en España (1954-2001), Tesis doctoral, Universidad Complutense de Madrid

Carcanholo, M.D. (2011). "El nuevo gobierno en Brasil y el legado de Lula: la continuidad de la política económica ortodoxa”, Revista Izquierda, 11 , 54-59.

CEPAL (2017). Anuario estadístico de América Latina y El Caribe, 2016, Comisiōn Económica para América Latina y El Caribe, Santiago de Chile. 
Del Rīo, A. (2018). Análisis centro-periferia en la UE-20 (1995-2014), Tesis doctoral, Universidad Complutense de Madrid.

FBBVA (2017): El stock y los servicios del capital en España y su distribución territorial y sectorial (1964-2014), Fundaciōn BBVA/Ivie, Madrid.

Feenstra, R., Inklaar, R. y Timmer, M. [PWT] (2015): "The Next Generation of the Penn World Table", American Economic Review, 105(10), 3150-3182. Disponible en <www.ggdc.net/pwt >

FMl (2017a): World Economic Outlook Database, Fondo Monetario Internacional, Washington DC, octubre.

FMI (2017b): International Financial Statistics, Fondo Monetario Internacional, Washington DC.

Freeman, A. (2004): "The Inequality of Nations", en Freeman, A. y Kagarlitsky, B. (eds.), The Politics of Empire. Globalisation in Crisis, Pluto Press, Londres.

IBGE (2017): Sistema de contas nacionais. Tabelas de recursos e usos, Instituto Brasileiro de Geografia e Estatística, Rio de Janeiro.

INE (2017): Contabilidad nacional anual de España. Base 2010, Instituto Nacional de Estadística, Madrid.

IPEA (2017): Base de dados macroeconômicos, Instituto de Pesquisa Econômica Aplicada, Río de Janeiro.

Marquetti, A. y Porsse, M. (2014): "Patterns of Technical Progress in the Brazilian Economy, 1952-2008”, CEPAL Review, 113, 57-73.

Martínez, J. (2011). "La estructura teórica Centro/Periferia y el análisis del sistema económico global: ¿obsoleta o necesaria?", Revista de Economía Mundial, 29, 29-59.

Martinnez-Hernāndez, F. (2017): "The Political Economy of Real Exchange Rate Behavior: Theory and Empirical Evidence for Developed and Developing Countries, 1960-2010", Review of Political Economy, 29(4), 566-596.

Mateo, J.P. (2007): La Tasa de ganancia en México, 1970-2003. Tesis doctoral, Universidad Complutense de Madrid.

Mateo, J.P. (2016): "The Great Recession in the US from the Perspective of the World Economy”, World Review of Political Economy, 7(2), 182-207.

Mateo, J.P. (2017): “Distorsiones y desequilibrios en la dinámica de acumulaciōn de la economía en España", Problemas del Desarrollo, 191 (48), 55-82.

Mateo, J.P. (2018): "The Accumulation of Capital and Economic Growth in Brazil. A long-term Perspective (1950-2008)”, Review of Radical Political Economics, 50(2), 370-391.

Mateo, J.P. (2020): "La acumulación de capital en la periferia. Una propuesta analítica desde la economía política”, Cuadernos de Economía, 43(122) (próxima publicaciōn).

Mateo, J.P., Montanyà, M. (2018): "The Accumulation Model of the Spanish Economy: Profitability, the Real Estate Bubble and Sectoral Imbalances, en L. Buendía y R. Molero (coords.): The Political Economy of Modern Spain: from Miracle to Mirage, Routledge, Londres (pp. 20-48). 
Morandi, L. (2015): "Estimativas do estoque de capital fixo para o Brasil: 1950-2014, Séries históricas", Instituto Brasileiro de Economia, Fundação Getulio Vargas (FGV-IBRE).

OCDE (2017): OECD Statistics, Organizaciōn Para la CoOperaciōn EConómica y el DesarRollo, Paris.

Palazuelos, E. (dir.) (2015): Economía política mundial, Akal, Madrid.

Saad-Filho, A. (2014): “The 'Rise of the South': Global Convergence at Last?, New Political Economy, 19(4), 578-600.

Shaikh, A. (2016): Capitalism: Competition, Conflict, Crises, Oxford University Press, Nueva York.

TCB (2015): Output, Labor and Labor Productivity, 1950-2015, The Conference Board Total Economy Database, septiembre.

Worth, O. y Moore, P. (eds) (2009): Globalization and the 'New' SemiPeripheries, Palgrave Macmillan, Basingstoke.

\section{Anexo. Cuestiones metodológicas}

Dada la definición proporcionada del stock de capital, se toman las series oficiales descontando las actividades financieras e inmobiliarias, junto a las Administraciones Públicas y servicios sociales. El stock de capital para el año $t\left(K_{t}\right)$ corresponde a $K_{t-1}$, el deflactor $P_{y}$ se toma del FMI (2017a), los TC de España y Brasil de OCDE (2017) y FMl (2017b), y los coeficientes de la PPA de FMI (2017a). En Brasil, el stock de capital se calcula enlazando la serie de Morandi (2015) a la estructura de la serie de IPEA (2017) a partir de 2008, para mantener así la homogeneidad con la base de precios. Para el empleo, se utiliza la serie de IBGE con la estructura por actividades de Marquetti y Porsse (2014) entre 1990 y 1999. 
